Document downloaded from:

http://hdl.handle.net/10251/147738

This paper must be cited as:

De Battista, H.; Picó-Marco, E.; Santos-Navarro, FN.; Picó, J. (2019). Output Feedback Linearization of Turbidostats After Time Scaling. IEEE Transactions on Control Systems Technology. 27(4):1668-1676. https://doi.org/10.1109/TCST.2018.2834882

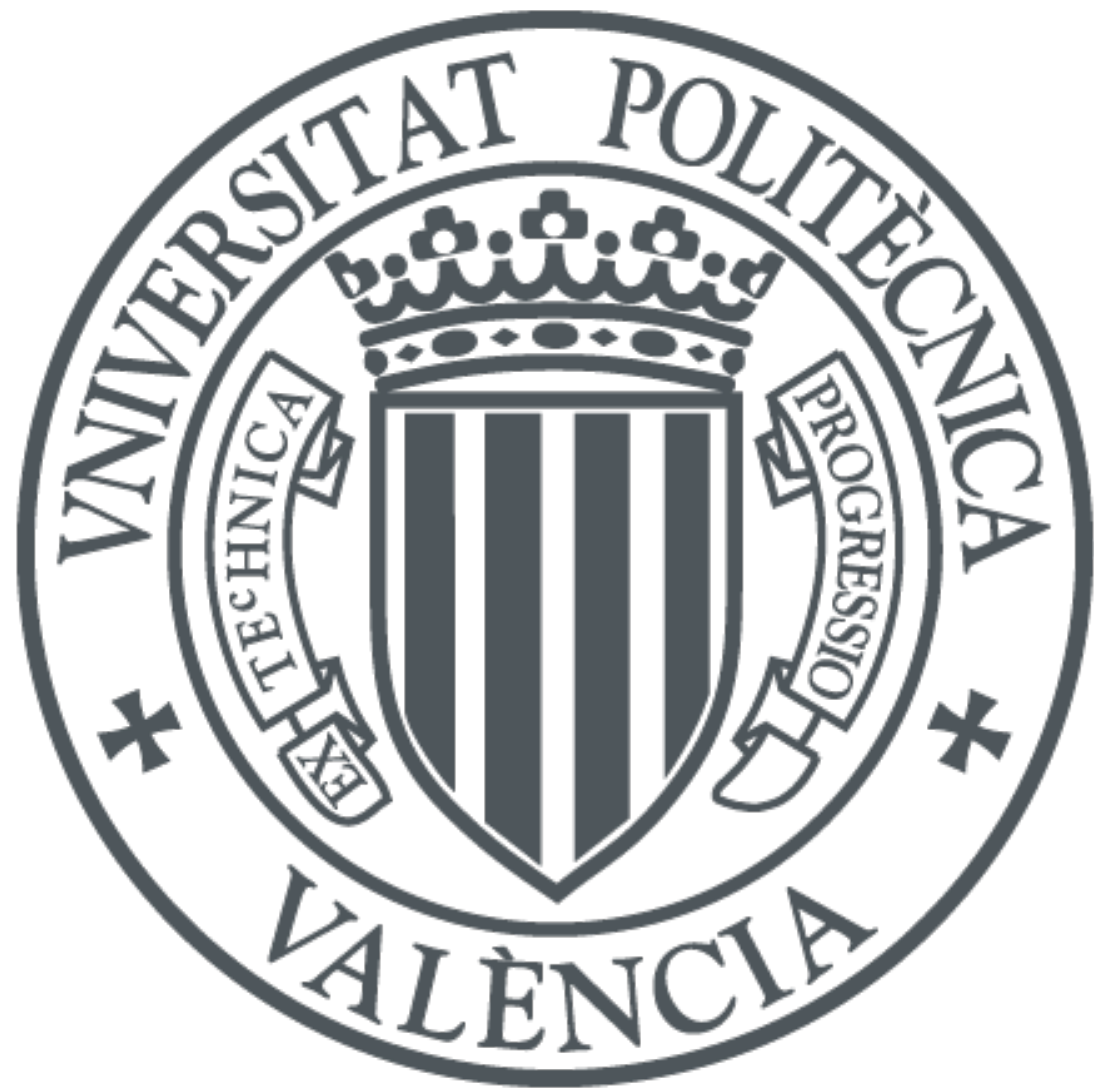

The final publication is available at

https://doi.org/10.1109/TCST.2018.2834882

Copyright Institute of Electrical and Electronics Engineers

Additional Information

"(C) 2019 IEEE. Personal use of this material is permitted. Permissíon from IEEE must be obtained for all other uses, in any current or future media, including reprinting/republishing this material for advertisíng or promotional purposes, creating new collective works, for resale or redistribution to servers or lists, or reuse of any copyrighted component of this work in other works." 


\title{
Output feedback linearization of turbidostats after time scaling
}

\author{
Hernán De Battista, Enric Picó-Marco, Fernando N. Santos-Navarro, and Jesús Picó, Senior Member, IEEE
}

\begin{abstract}
Turbidostats are a class of bioreactors gaining interest due to the recent availability of micro- and small-scale devices for characterization and scaling-up of biotechnological systems relevant in the biotech and pharma industries. The goal is to keep cell density constant in continuous operation. Thus the control law, i.e. the substrate feeding strategy, must guarantee global or semiglobal convergence to an equilibrium point. However, their control is difficult due to the uncertain, time-varying and nonlinear nature of the processes involved. In this paper we propose an adaptive control law that globally stabilizes the desired biomass set-point. Further, in a certain region of the state space the controller linearizes the dynamic behavior after some time scaling. This way, the orbits of the closed loop system are imposed by the designer. The intrinsic integral action of the gain adaptation rejects parameter uncertainties. Moreover, the controller implementation only assumes biomass concentration to be measured. Both simulated and experimental results show the performance of the controller.
\end{abstract}

Index Terms-Bioreactors; Nonlinear control systems; Output feedback; Adaptive control.

\section{INTRODUCTION}

$\mathbf{I}$ NDUSTRIAL biotechnology uses enhanced and/or genetically modified microorganisms to produce specialty metabolites (e.g. amino acids, vitamins, food additives, biofuels,...) of importance for the health, chemical, food and energy sectors among others. Bioreactors are the workhorses where characterization, scaling-up, and production take place. Therefore, their feedback control has received much attention. However, control of bioreactors is difficult due to: (i) uncertainty on key variables of the system representing the physiological state of the culture, (ii) non-linear process dynamics, and (iii) large variability. In this context, modelbased design of controllers has been addressed using simple models based on mass-balances [1] and developing generic and robust controllers based on the minimal model concept [2][4]. On the one hand, mass-balance based models concentrate the uncertainty in specific terms; the bioreaction kinetics, and the bioreaction yields. On the other, robust controllers based on the minimal model concept use the mass-balance structure and rough information on the kinetics structure and bounds.

This work was supported by the National University of La Plata (11I127), ANPCyT (PICT2014 2394), CONICET (PIP112 2015 0837); and by MINECO/AEI/FEDER,UE (DPI2014-55276-C5-1-R, DPI2017-82896-C21-R). F.N. Santos-Navarro was granted by ai2-UPV. Corresponding author: jpico@ai2.upv.es

H. De Battista (deba@ing.unlp.edu.ar) is with the CONICET. LEICI, Facultad de Ingeniería, Universidad Nacional de La Plata, La Plata 1900, Argentina.

E. Picó-Marco, F. N. Santos-Navarro and J. Picó (\{enpimar, fersann1, jpico\}@upv.es) are with the Institut ai2, Universitat Politècnica de València, València, E46022, Spain.
In continuous bioreactions the volume of culture inside the bioreactor is kept constant by setting the inlet flow rate equal to the outlet one. The higher risk of contamination and cell mutation in continuous bioreactions have favoured its use only for processes involving microorganisms with high mutationstability. Yet continuous bioreactors have some advantages, like increased productivity for biomass and growth associated products, reduction of raw materials, waste production and maintenance requirements [5], or the possibility of analyzing cultures under sets of steady state conditions [6], [7]. Thus, industrial bioprocesses like biofuels, pharmaceutics and bioplastics production, etc. are increasingly being migrated to continuous bioreactors [8]. A few classes of continuous bioreactors are mostly used. In chemostats the goal is to keep a desired specific growth rate of the microorganism [8]. Nutristats are used to drive substrate concentration to a desired set-point, most often corresponding to optimal biomass or production rate [4], [9]. Several approaches have been used to stabilize the system at this optimum operating point, assuming measurement of full state [10]-[12], biomass [3], or reaction rate and substrate measurement or estimation [13]-[15].

Turbidostats regulate cell density at a prescribed value. Low cost micro- and small-scale turbidostats are increasingly becoming available for characterization and scaling-up of systems without nutrient limitation [16]-[21]. Cell density is continuously monitored either using a spectrophotometer/turbidometer to measure optical density [22], [23], or using methods based on dielectric permitivity [24], [25]. The control law, i.e. the substrate feeding strategy, must guarantee global or semiglobal convergence of biomass to an equilibrium point.

Linear control theory was used in [26], and exact feedback linearisation in [10], [27]-[29]. Feeding strategies that are proportional to the reaction rate avoid biomass washout and also avoid falling in batch operation. Variations adapting the controller gain [14], [30] or including error feedback [2], [31] have been proposed to achieve robustness against process uncertainties and variability, and to improve the transient response. In the first case, globally stable adaptive control laws that in the end are integral control ones are designed [14], [32], being the feedback gain dynamically adapted in such a way that control never saturates. Controllers using this idea can be applied to regulate biomass, substrate or product concentration in continuous bioprocesses. These controllers eliminate steady state errors but they were not designed to set the transient response by controller tuning. To speed up the transient, a nonlinear proportional control with adaptive gain leading to a class of nonlinear PI controller was proposed in [2] to control growth rate. Though exhibiting fast convergence properties and robustness, it is not clear how to impose the 
desired dynamics and care should be taken to avoid controller saturation. A saturated PI control is proposed in [3] with implicit anti-windup protection, achieving robust closed-loop stability. Biomass is measured and substrate is estimated using an observer. The gains of both observer and controller must meet some bounding conditions, and an iterative tuning procedure is proposed to set them.

In this paper we propose an adaptive control law that globally stabilizes the desired biomass concentration. Time scaling and Stability Preserving Maps are used as tools to simplify stability analysis and controller design [33]. The controller linearizes the dynamic behavior in a certain region of the state space after time scaling. This way, the closed loop orbits are imposed by the designer, resulting in very simple tuning rules. Neither a detailed model of the growth kinetics nor knowledge of the bioreaction yields are required. The controller assumes biomass concentration is measured and the reaction rate is estimated from it using high-gain or sliding observers [33], [34] whereby a practical principle of separation can be assumed. The intrinsic integral action of the gain adaptation rejects parameter uncertainties. In case the reaction rate is indirectly measured or calculated with some error then the steady state output error is bounded.

The paper is organized as follows. In Section II the problem is formulated. The proposed control law and its analysis are considered in Section III and Section IV respectively. Simulations highlighting the performance of the controller are shown in Section $\mathrm{V}$ and its experimental validation in Section VI. Conclusions are outlined in Section VII.

\section{Problem Statement}

Consider a pure culture growing under a single substrate being continuously fed to the reactor [1], [26]:

$$
\begin{aligned}
\dot{x} & =\mu(x, s, \mathbf{q}) x-D(t) x \\
\dot{s} & =-y \mu(x, s, \mathbf{q}) x+D(t)\left(s_{i}-s\right) \\
\dot{\mathbf{q}} & =Q(\mathbf{q}, x, s, D(t))
\end{aligned}
$$

where $x \in \Re_{+}$is the biomass concentration, $s \in \Re_{+}$the substrate concentration, $s_{i} \in \Re_{+}$the substrate concentration in the inlet flow, $y$ the substrate-biomass conversion yield, $D(t)$ the dilution rate, i.e. the ratio between the flow rate and the culture volume that will be used as control input, and $\mu(x, s, \mathbf{q})$ the specific growth rate, where $\mathbf{q}$ gathers uncertain parameters and other variables ( $\mathrm{DO}$, temperature, $\mathrm{pH}$, growthlinked products, etc.) affecting the reaction kinetics.

Model (1) encompasses a large number of practical cases for production of biomass and growth-linked products.

Assumption 2.1: Assume the specific growth rate vanishes if there is no substrate $(\mu(x, 0, \mathbf{q}) \equiv 0)$, is strictly positive whenever substrate is available $(\mu(x, s, \mathbf{q})>0 \quad \forall s>0)$, and is bounded $\left(\mu(x, s, \mathbf{q}) \leq \mu_{m} \quad \forall x, s, q \geq 0\right)$. For technical reasons, assume it can be modeled by a continuously differentiable function of $x$ and $s\left(\mu(x, s, \mathbf{q}) \in \mathcal{C}^{1}\right)$.

This assumption is fulfilled by all standard specific growth rate models [1].

Assumption 2.2: Assume biomass concentration $x$ is available for measurement.
Definition 2.1: A bioprocess operates in continuous mode when $D(t) \geq d>0 \forall t \geq t_{0}$ for some sufficiently small $d$.

The goal is to design a control law $D(t)$ that globally stabilizes system (1) at the specified biomass set-point $x^{*}$.

\section{PROPOSED CONTROL LAW}

\section{A. Growth rate proportional feeding law}

Consider a controller of the form

$$
D(t)=\gamma \mu(t) x
$$

In the following we use $\mu(t)$ to emphasize the time varying nature of the specific growth rate and that the feedback law is not constructed from the uncertain model $\mu(x, s, \mathbf{q})$ but from an observer-based estimation.

Using (2), the closed loop dynamics for biomass and substrate can be expressed as:

$$
\begin{aligned}
& \dot{x}=D(t)\left(\gamma^{-1}-x\right) \\
& \dot{s}=D(t)\left(s_{i}-y \gamma^{-1}-s\right)
\end{aligned}
$$

Consider the simplest case, with $\gamma=\gamma^{*} \triangleq 1 / x^{*}$. Then:

$$
\begin{aligned}
\dot{x} & =D(t)\left(x^{*}-x\right) \\
\dot{s} & =D(t)\left(s^{*}-s\right)
\end{aligned}
$$

where $s^{*}=s_{i}-y x^{*}$ is the substrate equilibrium point. It is clear that for a continuous bioreaction as stated in Definition 2.1, the controller (2) with $\gamma=\gamma^{*}$ locally stabilizes the equilibrium $P^{*}=\left(x^{*}, s^{*}\right)$ of (4).

\section{B. Time scaling}

The idea now is to semiglobally stabilize the biomass concentration preserving the control law structure (2), i.e. to feed the reactor in proportion to growth rate, but shaping the feedback gain $\gamma$ so as to improve the transient dynamics. To this end, it is convenient to express the closed loop dynamics in a new time scale $\tau$ where the controller design problem becomes simpler. Consider the time scaling given by:

$$
d \tau=D(t) d t
$$

where $\tau$ can be interpreted as the volume of substrate fed in $\left[t_{0}, t\right]$ relative to the bioreactor one. Now, defining the time derivative w.r.t. the new time variable as $(\cdot)^{\prime}=\frac{d(\cdot)}{d \tau}$, the closed loop dynamics (3) become:

$$
\begin{aligned}
& x^{\prime}=\gamma^{-1}-x \\
& s^{\prime}=s_{i}-y \gamma^{-1}-s
\end{aligned}
$$

Notice the system in the transformed time scale has its eigenvalues at -1 , and the parameter $\gamma$ can be shaped to improve convergence of one variable, say $x$, relative to the other. Notice also that the dynamics of biomass and substrate are decoupled in the transformed time scale. 


\section{Adaptive shaping of $\gamma$}

The goal now is to shape $\gamma$ so as to improve convergence of $x$ towards the desired set-point $x^{*}$. To this end, we propose the following adaptive control law in the $\tau$-scale:

$$
\gamma^{\prime}=-\gamma^{2}\left[\left(\gamma^{-1}-x\right)(1-a)-b\left(x-x^{*}\right)\right] f(\gamma)
$$

where $f(\gamma)$ is a saturation function satisfying:

$$
\begin{array}{ll}
f \in \mathcal{C}^{1} & \\
f(\gamma)>0 & \forall \gamma \in(\underline{\gamma}, \bar{\gamma}), \quad \underline{y}<\underline{\gamma}<\gamma^{*}<\bar{\gamma} \\
f(\gamma)=1 & \forall \gamma \in \Gamma=\left[\gamma_{m}, \gamma_{M}\right] \subset(\underline{\gamma}, \bar{\gamma}), \quad \gamma^{*} \in \Gamma \\
f(\gamma)=0 & \forall \gamma \notin(\underline{\gamma}, \bar{\gamma}) .
\end{array}
$$

and $\gamma_{0}=\gamma\left(\tau_{0}\right) \in(\underline{\gamma}, \bar{\gamma})$.

To prove global stability of the closed loop system corresponding to the biomass dynamics define the errors:

$$
\begin{aligned}
& \tilde{x}=x-x^{*} \\
& \tilde{\gamma}=\gamma^{-1}-\gamma^{*-1}
\end{aligned}
$$

Taking derivatives w.r.t. $\tau$ we obtain the error dynamics

$$
\begin{aligned}
& \tilde{x}^{\prime}=\tilde{\gamma}-\tilde{x} \\
& \tilde{\gamma}^{\prime}=f(\gamma)[(\tilde{\gamma}-\tilde{x})(1-a)-b \tilde{x}]
\end{aligned}
$$

Let us now define the following positive definite radially unbounded function:

$$
\begin{aligned}
W_{1} & =\int_{0}^{\tilde{\gamma}} \frac{g}{f\left(\frac{\gamma^{*}}{1+g \gamma^{*}}\right)} d g \\
W_{2} & =\frac{b-a+1}{2} \tilde{x}^{2} \\
W & =W_{1}+W_{2}
\end{aligned}
$$

with $b-a+1>0$. Using the Leibnitz Integral Rule for $W_{1}$, the $\tau$-time derivative of (11) along the state trajectory is:

$$
\begin{aligned}
& W_{1}^{\prime}=\frac{\tilde{\gamma} \tilde{\gamma}^{\prime}}{f(\gamma)}=-(a-1) \tilde{\gamma}^{2}-(b-a+1) \tilde{\gamma} \tilde{x} \\
& W_{2}^{\prime}=-(b-a+1) \tilde{x}^{2}+(b-a+1) \tilde{\gamma} \tilde{x} \\
& W^{\prime}=-(a-1) \tilde{\gamma}^{2}-(b-a+1) \tilde{x}^{2}
\end{aligned}
$$

which is negative definite for $a>1$. Therefore, the equilibrium $(\tilde{x}, \tilde{\gamma})=(0,0)$ of the error dynamics (10) is globally asymptotically stable.

Next we show that, after some finite time $T c$ the control law (2)-(7) linearizes the biomass dynamics in the $\tau$-scale within the set $\Gamma=\left[\gamma_{m}, \gamma_{M}\right]$. To this end, differentiating $x$ twice w.r.t. $\tau$ in (6) one gets

$$
\begin{aligned}
x^{\prime \prime} & =-\gamma^{-2} \gamma^{\prime}-x^{\prime} \\
& =f(\gamma)\left[x^{\prime}(1-a)-b\left(x-x^{*}\right)\right]-x^{\prime}
\end{aligned}
$$

Since $\tilde{\gamma}$ asymptotically converges to zero, there will exist some finite time $T_{c}$ such that $\gamma \in \Gamma$ for all $\tau \geq T_{c}$ where, from (8), $f(\gamma)=1$. Therefore, once $\gamma$ enters the set $\Gamma$ the system will behave according to the linearised dynamics:

$$
x^{\prime \prime}+a x^{\prime}+b\left(x-x^{*}\right)=0
$$

Remark 3.1: Notice that although in this linear region in the $\tau$-scale it suffices to choose $a, b>0$ to ensure asymptotic stability, the Lyapunov function (11) we found is more restrictive.

\section{Stability in the original time scale}

In the original time scale $t$, the control law becomes:

$$
\begin{aligned}
D(t)= & \gamma \mu(t) x \\
\dot{\gamma}=- & \gamma^{2}\left[(\mu(t)-D(t)) x(1-a)-D(t) b\left(x-x^{*}\right)\right] f(\gamma) \\
& \quad \gamma_{0} \in(\underline{\gamma}, \bar{\gamma})
\end{aligned}
$$

with $b>(a-1)>0$ and $f(\gamma)$ defined as in (8).

Stability of the system (3)-(15) is equivalent to that of (6)(7) if the time scaling (5) defines a stability preserving map.

Theorem 3.1: If $D(t)$ is bounded and strictly positive, the time scaling (5) defines a stability preserving map.

Proof If $D(t)$ is bounded and strictly positive, the time-scaling (5) defines a strictly increasing and onto function $\xi: t \longrightarrow \tau$. Thus, the homomorphism given by the identity transformation for the coordinates and the time-scaling defined by (5) preserves stability. See Th. 7 and Coroll. 8 in [33].

Now we must prove that the dilution rate $D(t)$ in (15) is bounded and strictly positive. That is, we must prove that the control law (15) induces continuous mode operation.

First we prove that $D(t)$ does not vanish. The saturation function (8) in the adaptive control law (15) ensures that

$$
\gamma(t) \in(\underline{\gamma}, \bar{\gamma})>0 \quad \forall t \geq t_{0}
$$

Therefore, it will suffice to prove that both biomass $x(t)$ and the specific growth rate $\mu(t)$ are bounded away from zero. That is, we must prove that the control law avoids both washout of biomass and batch operation with substrate depletion.

Notice $(x=0, s, q)$ are stable fixed points of system (1) which correspond to biomass washout. We can assume initial conditions satisfying $x_{0}>0$, i.e. there is some initial biomass concentration in the bioreactor. To avoid washout the region $R_{\overline{\mathcal{X}}}=\{(x, s, q) \mid x \geq \underline{x}\}$ must be positively invariant for some sufficiently small $0<\underline{x}<x^{*}$. By continuity $R_{\overline{\mathcal{X}}}$ will be locally non-attractive if the dilution $D(t)$ is smaller than the specific growth rate as the biomass approaches zero:

$$
\lim _{x \rightarrow 0^{+}} D(t)=\lim _{x \rightarrow 0^{+}} \gamma(t) \mu(x, s, q) x<\mu(0, s, q)
$$

Taking into account (16), condition (17) is trivially fulfilled. Thus, the control law (15) avoids washout.

Now, given the properties for the specific growth rate in Assumption 2.1 and the result above, to prove that the specific growth rate $\mu(x, s, q)$ does not vanish it suffices to prove that the substrate concentration $s$ so does not, i.e. the control law avoids batch operation with substrate depletion. From the second equation in (1), it will suffice to show that

$$
\lim _{s \rightarrow 0^{+}} D(t)=\lim _{s \rightarrow 0^{+}} \gamma(t) \mu(x, s, q) x>y x \lim _{s \rightarrow 0^{+}} \frac{\mu(x, s, q)}{s_{i}-s}
$$

so that $\mathcal{S}=\{(x, s, q) \mid s=0\}$ is locally non-attractive. Notice the control law (15) fulfills condition (18) provided $\underline{\gamma}>\frac{y}{s_{i}}$, as required in the definition (8) of the saturation function $f(\gamma)$. Therefore, the specific growth rate $\mu(x, s, q)$ is bounded away from zero.

Finally, we prove upper boundedness of the dilution rate $D(t)$. Assumption 2.1 and (16) ensure both the specific growth rate $\mu(t)$ and the adaptation gain $\gamma$ are upper bounded. So, it 
only remains to show that biomass $x$ is also upper bounded. From (3), positivity of $D(t)$, and boundedness of $\gamma(t)$ given in (16), the biomass concentration $x$ cannot grow unboundedly for its derivative will be negative whenever $x>\gamma^{-1}$.

Thus, we have proved that the dilution rate $\bar{D}(t)$ is both bounded from above and bounded away from zero. Therefore, the time scaling (5) is well defined.

Notice $D$ vanishes on $\mathcal{S}$. Therefore, the controller is not able to start the process from $s=0$. Anyway, the non-attractiveness condition (18) is always satisfied since $x^{*}<\frac{s_{i}}{y}$. Thus, it (semi)globally stabilizes the equilibrium.

\section{Controller tuning}

The key tuning parameters of the controller are the gains $a$ and $b$. Some guidelines are given below.

\section{A. Closed loop poles in the $\tau$-time scale}

Recall that after some finite time, the biomass error in the transformed time scale $\tau$ will follow the linear dynamics (14). By choosing $a, b$ in the control law (15), and using $b-a+1>$ 0 , we can place the corresponding eigenvalues $\lambda_{1}, \lambda_{2}$ of the closed-loop system with:

$$
\begin{aligned}
\omega_{0} & =\sqrt{\lambda_{1} \lambda_{2}}=\sqrt{b} \\
\xi & =\frac{\lambda_{1}+\lambda_{2}}{2 \omega_{0}}=\frac{a}{2 \sqrt{b}} \\
\frac{1}{2} \omega_{0}^{-1} & <\xi<\frac{1}{2}\left(\omega_{0}+\omega_{0}^{-1}\right)
\end{aligned}
$$

where $\omega_{0}$ is the natural frequency, $\xi$ the damping coefficient. Equivalently, the last inequalities can be rewritten in terms of $\xi$ and the damping factor $\sigma=\xi \omega_{0}$ as

$$
\sigma>\frac{1}{2} \quad \xi<\frac{\sigma}{\sqrt{2 \sigma-1}}
$$

\section{B. Effects of the growth rate observer}

Any biased measurement in $x$ will produce an error at steady state independently of the controller tuning. To analyze the controller performance we consider that estimation $\hat{\mu}$ of the specific growth rate $\mu$ introduces an error, so that $\Delta \mu=(\mu-$ $\hat{\mu}) / \mu$. Then (1) and (15) become:

$$
\begin{aligned}
& \dot{x}=\gamma \mu(t) x\left(\gamma^{-1}-\frac{\hat{\mu}(t)}{\mu(t)} x\right) \\
& \dot{\gamma}=-\gamma^{2} \hat{\mu}(t) x\left[(1-\gamma x)(1-a)-\gamma b\left(x-x^{*}\right)\right] f(\gamma)
\end{aligned}
$$

that evaluated at steady state result in:

$$
x_{s s}=\frac{x^{*}}{1-\frac{(a-1)}{b} \Delta \mu}
$$

The effect of error in estimation of the specific growth rate will depend on the term:

$$
\Delta_{x / \mu}:=\frac{(a-1)}{b}
$$

Recall that $b>a-1>0$ and $a>1$, and notice $\Delta_{x / \mu} \rightarrow 0$ as $a \rightarrow 1$. Therefore, there is a compromise between transient response (speed and overshoot) and steady state error. If $a, b$ are designed to have a given damping coefficient $\xi$ and damping factor $\sigma=a / 2$ (thus a given settling time), then

$$
\Delta_{x / \mu}=\frac{(2 \sigma-1) \xi}{\sigma^{2}}
$$

For a given $\xi$, the error has a single local maximum $\Delta_{x / \mu, \max }=\xi$ at $\sigma=1(a=2)$ and goes to zero as $a \rightarrow 1$ or $a \rightarrow \infty$. Also, the smaller $\xi$, the smaller the error will be. The steady state error of the classical algorithm (2) is very similar to the error in the estimation of $\mu: \Delta_{x / \mu} \cong 1$. Thus, if well designed, the proposed controller will significantly reduce the error. Anyway, to avoid this source of error one can estimate $\mu$ using a supertwisting observer that gives finite time convergence with zero steady state estimation error [33].

\section{Design of $f(\gamma)$}

The span of $\gamma$ is constrained for the restriction $\frac{y}{s_{i}}>\gamma$ and by the maximum flow rate of the pumping system. The inequality $\underline{\gamma}>\frac{y}{s_{i}}$ implies that the desired set-point for biomass concentration must be bounded by $x^{\star}<\frac{s_{i}}{y}$, just expressing that the amount of substrate converted into biomass at equilibrium $\left(y x^{\star}\right)$ must be smaller than the one in the input flow $\left(s_{i}\right)$. The adaptation limits $\underline{\gamma}$ and $\bar{\gamma}$ must be set to fulfill these constraints. The limits of the linear region $\gamma_{m}$ and $\gamma_{M}$ can be close to $\underline{\gamma}$ and $\bar{\gamma}$ but considering that a smooth transition from 1 to 0 is required.

\section{Simulated Results}

Intensive simulation analysis has been carried out to verify the main features of the proposed control law. Set-point changes, process start-up and effect of the initial conditions have been evaluated considering growth kinetics without and with substrate inhibition. Furthermore, the control strategy has been compared with previous proposals in the literature, and the advantages and disadvantages are discussed. The process dynamics (1) has been simulated using the specific growth kinetics plotted in Fig. 1a and process parameters $y=2.22$ and $s_{i}=3.6$. The dilution rate is given by the dynamic feedback law (15), with bounding function $f(\gamma)$ as depicted in Fig. 1b. The tuning constants $a, b$ were set to different values. In this section, the growth rate is assumed to be known so as to avoid including observer dynamics in the controller assessment.

The controller performance has been evaluated in time scales $t$ and $\tau$ since both lead to interesting conclusions. On the one hand, the $t$-scale measures the process time in hours having an obvious meaning. On the other, the time unit in the $\tau$-scale is the retention time, so that $\tau$ represents the times the volume of the bioreactor is entirely exchanged. That is, the duration of the transient response in the $\tau-$ scale represents the amount of medium consumed before reaching the new setpoint. A key advantage of the $\tau-$ scale is that the transient is independent of the dilution rate, which in steady state equals the specific growth rate. On the other hand, processes with lower specific growth rates are slower in the $t$-scale, thus controllers performance is more difficult to compare. It is important to point out also that the time mapping $t(\tau)$ is monotonous and preserves the amplitude of a time response. Moreover, as a general rule, faster responses in the $\tau$-scale are typically faster in the $t$-scale also. 

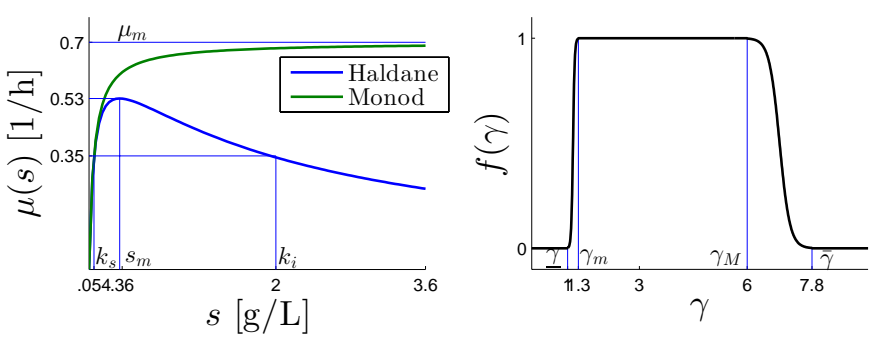

Figure 1. (a) Specific growth kinetics $\mu(s)$. (b) Bounding function $f(\gamma)$ of controller (15).

\section{A. Set-point change}

The response of the process to a set-point change for different controller settings is first presented. The initial operating point is $\left(x_{0}, s_{0}, \gamma_{0}\right)=\left(1 / 5, s_{i}-y x_{0}, x_{0}^{-1}\right)$ and the final one is $\left(x^{*}, s^{*}, \gamma^{*}\right)=\left(1 / 3, s_{i}-y x^{*}, x^{*-1}\right)$. The controller parameters $a$ and $b$ were chosen so that the damping coefficient is constant $(\xi=.5)$ while $\omega_{0}$ ranges from 2 to 12 .

Fig. 2 and Fig. 3 show the results for Monod and Haldane specific growth kinetics. Note that the overshoot in the $x$-response is the same for all $\omega_{0}$ but $\omega_{0}=12$. The reason is that the controller temporarily leaves its linear region in this case (see the plot of $\gamma$ ), so the expected linear behavior (14) is temporarily lost. In case the controller always operates in its linear region, there is a direct mapping between $(a, b)$ and the response in the $\tau$-scale. Because of the nature of the time scaling, the underdamped response designed in the $\tau$ domain is also observed in the $t$-scale. Moreover, as observed in the figures, the order is also preserved. The faster responses in the $\tau$-scale are also the faster ones in the $t$-scale. The reason is that, although the $t(\tau)$ mappings are not the same for all cases, they are very similar. Moreover, at the peak or settling time of a given response, the $t(\tau)$ mapping practically coincides with the ones corresponding to faster responses.

From Fig. 2 and Fig. 3 note that in the $t$-scale the process with inhibition is slower than without it even if the responses in the $\tau$-scale are equal, being consistent with the fact that the dilution rate is lower. Note also that the shape of the $t(\tau)$ mappings are very similar for both growth kinetics.

\section{B. Start-up}

The process has been also simulated for the same setpoint as before but starting from very low initial conditions $\left(x_{0}, s_{0}, \gamma_{0}\right)=\left(.05, .01, x^{*}\right)$. Note that the process is not initially at steady state. Fig. 4 and Fig. 5 show the results for the Monod and Haldane growth kinetics and for different controller tunings. In particular, the response for three different damping coefficients $\xi$ and two different damping factors $\sigma=\xi \omega_{0}$ are depicted. The responses in the $\tau$-scale present the classical linear second-order dynamics while $\gamma$ keeps between $\left(\gamma_{m}, \gamma_{M}\right)$. The responses in the $t$-scale are very similar, preserving amplitude and order. Comparing both figures, notice the $x$ responses in the $\tau$-scale are identical, whereas the responses in the $t$-scale are very similar, although the process with Monod kinetics is a bit faster because the specific growth rate is higher. This shows the robustness of the controller with respect to the growth rate kinetics.
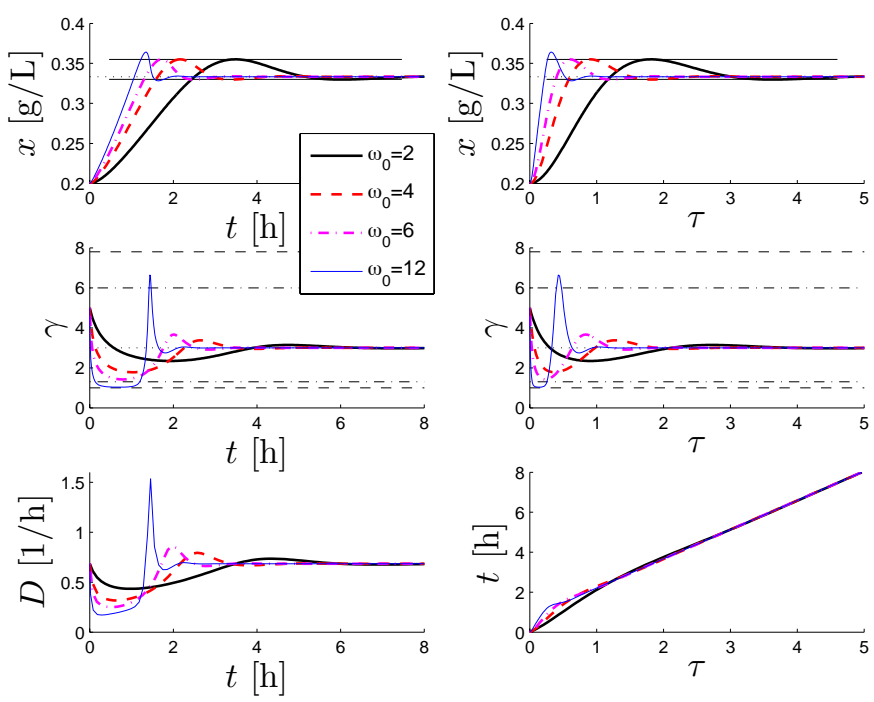

Figure 2. Set-point change for Monod kinetics. Former set-point: $\left(x_{0}, s_{0}, \gamma_{0}\right)=(.2,3.16,5)$, new set-point: $\left(x^{*}, s^{*}, \gamma^{*}\right)=(1 / 3,2.86,3)$.
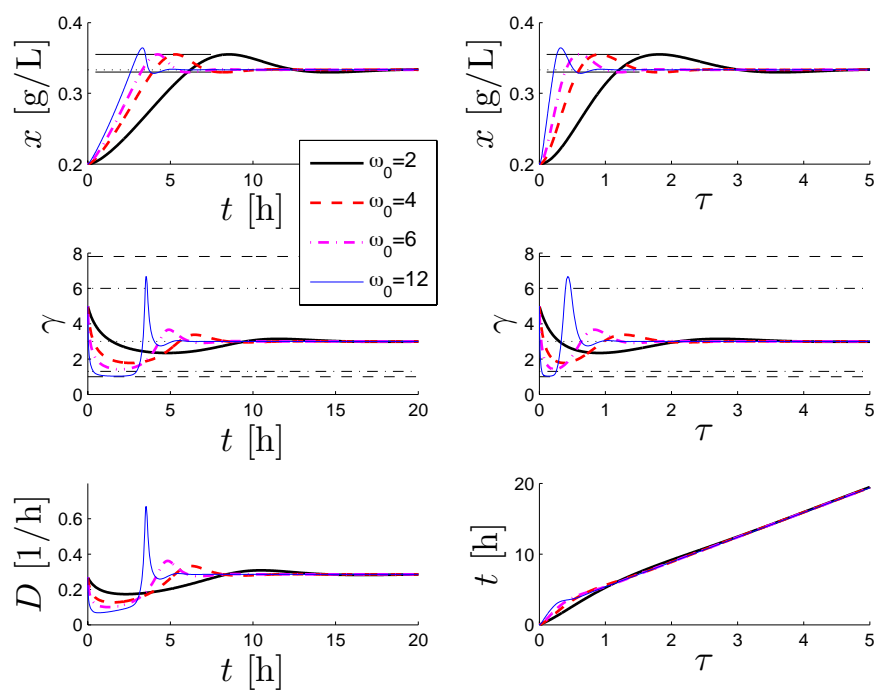

Figure 3. Set-point change for Haldane kinetics. Former set-point: $\left(x_{0}, s_{0}, \gamma_{0}\right)=(.2,3.16,5)$, new set-point: $\left(x^{*}, s^{*}, \gamma^{*}\right)=(1 / 3,2.86,3)$.

In addition, the controller performance is robust with respect to variability in the substrate initial conditions, as observed in Fig. 6. This figure shows the start-up from different initial substrate concentrations for Monod kinetics and setting $\sigma=$ $1.5, \xi=.75$, evidencing that subsystem $x-\gamma$ is independent of the substrate initial condition in the $\tau$-scale. Therefore, only the dependence of the time-scaling on the substrate $s$ when getting back into the original time scale $t$-via the specific growth rate $\mu(x, s)$ - will induce differences in the $t$-scale. Anyway, the time responses of biomass preserve the shapes and amplitudes of the responses in the $\tau$-scale.

\section{Comparison with other controllers}

Next the proposed controller is compared with the baseline controller $D(t)=\gamma^{*} \mu(t) x$ and with the one proposed in [14]:

$$
\begin{aligned}
\dot{\gamma} & =K \mu(t) x\left(x-x^{*}\right)(\gamma-\underline{\gamma})(\bar{\gamma}-\gamma) \quad \gamma_{0} \in(\underline{\gamma}, \bar{\gamma}) \\
D(\mu, x) & =\gamma \mu(t) x
\end{aligned}
$$



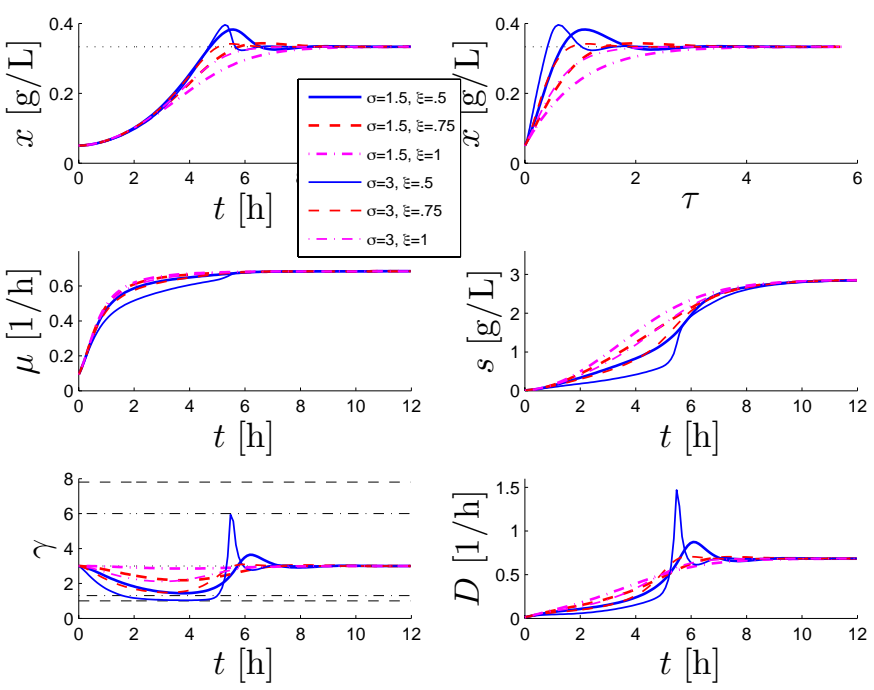

Figure 4. Process start-up for Monod kinetics. Initial condition: $\left(x_{0}, s_{0}, \gamma_{0}\right)=(.05, .01,3)$, set-point: $\left(x^{*}, s^{*}, \gamma^{*}\right)=(1 / 3,2.86,3)$.
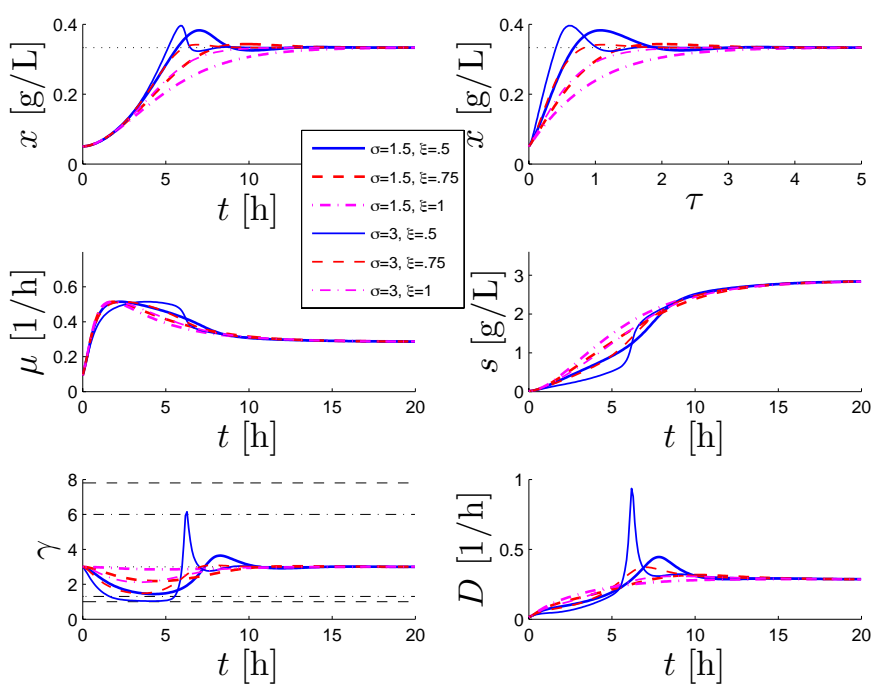

Figure 5. Process start-up for Haldane kinetics. Initial condition: $\left(x_{0}, s_{0}, \gamma_{0}\right)=(.05, .01,3)$, set-point: $\left(x^{*}, s^{*}, \gamma^{*}\right)=(1 / 3,2.86,3)$.
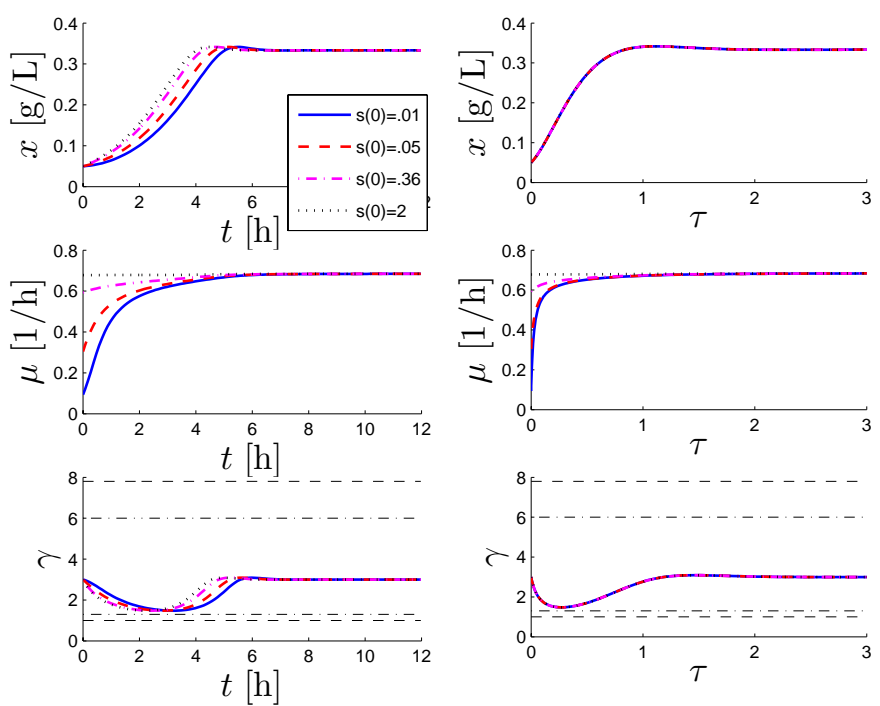

Figure 6. Process start-up from different initial substrate concentrations for Monod kinetics. Initial condition: $\left(x_{0}, s_{0}, \gamma_{0}\right)=\left(.05, s_{0}, 3\right)$, set-point $\left(x^{*}, s^{*}, \gamma^{*}\right)=(1 / 3,2.86,3)$. Controller tuning: $(\xi, \sigma)=(.75,3)$.
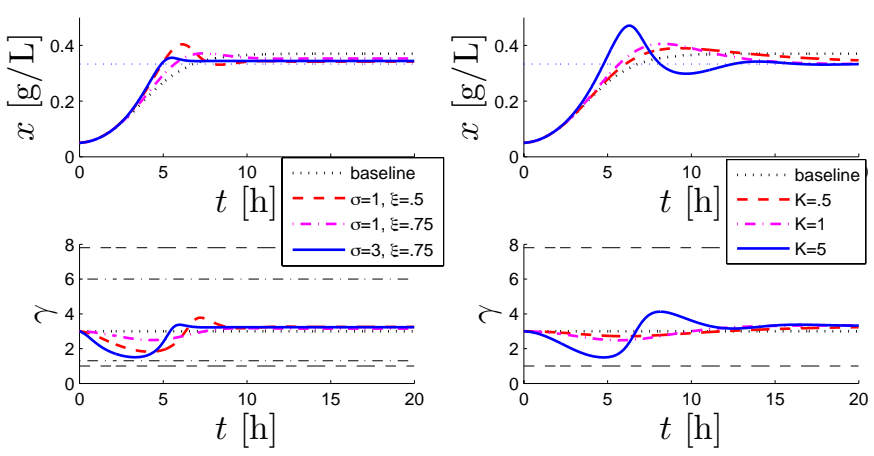

Figure 7. Effect of $\mu$ estimation error on controller performance using the proposed (left) and (25) (right) controllers. Initial condition: $\left(x_{0}, s_{0}, \gamma_{0}\right)=$ $(.05, .01,3)$, set-point: $\left(x^{*}, s^{*}, \gamma^{*}\right)=(1 / 3,2.86,3)$.

Notice controller (25) consists basically of the baseline controller where the gain $\gamma$ is adapted, introducing an integral compensation. The main feature of this controller is its capability to reject disturbances at steady state. However, since it has only one tuning parameter and it does not include proportional action, its potential to improve the transient response is limited. In fact, the cost of faster responses is larger overshoots. This is probably the main shortcoming of this approach. On the other hand, the controller proposed in this paper allows improving the desired transient response and is very easy to tune. Yet, although significantly attenuated, some measurement errors cannot be completely rejected.

Fig. 7 shows the time $t$ evolution of biomass concentration and adaptation gain for different tunings of the proposed controller (left) and controller (25) (right) in presence of a $-10 \%$ error in the estimation of $\mu(t)$. The response obtained with the baseline controller is plotted in both columns. The simulation scenario of start-up is repeated. Notice with the proposed controller one can achieve better settling time and can easily set the desired transient specifications. This is because there are two tuning parameters to set the linear second order dynamics (in $\tau$-scale). Furthermore, it significantly reduces the error in comparison with the baseline controller as predicted by (24) form $11 \%$ to $2.5 \%$. On the other hand, controller (25) achieves zero error thanks to the integral action. Yet, in pure integral adaptive controllers as (25), a large integral action that drives the error to zero reasonably fast deteriorates significantly the transient response. Although controller (25) response in the large tends to the desired value, the transient is longer (12 hours) than the one obtained with our controller (5 hours). These results suggest exploring the possibility of combining or scheduling both controllers in order to improve the transient response and completely reject steady state errors.

\section{EXPERIMENTAL RESULTS}

We used the experimental setup shown in Fig. 8. It consists of a $16 \mathrm{ml}$ turbidostat adapted from [17] and fed by a syringe pump (NE-1000, New Era Pump Systems, Inc.). Volume was kept constant by injecting pressurized air using a small pump. Optical density at $650 \mathrm{~nm}\left(\mathrm{OD}_{650}\right)$ was measured with an absorbance custom-made sensor using a fotodiode converting light intensity to frequency (TSL235-LF, Farnell). In our 


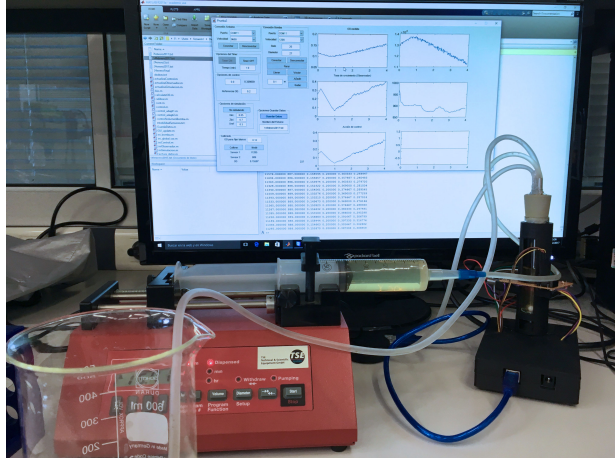

Figure 8. Experimental turbidostat setup.

working range there was a linear relationship between $\mathrm{OD}_{650}$ and biomass concentration, with $O D_{650}=1$ approximately corresponding to $1.5 \mathrm{~g} / \mathrm{L}$ wet weight, so we controlled optical density. We grew E. coli transformed cells containing the bluepurple chromoprotein amilCP using SOB medium as substrate with $20 \mathrm{mM}$ glucose $(3.603 \mathrm{~g} / \mathrm{L})$. We expected a yield for $E$. coli grown on glucose around $y=2.2 \mathrm{~g}$ glucose/g biomass [35] so that $y / s_{i} \approx 0.6$. Therefore we used the lower bound $\underline{\gamma}=1$ with the saturation function (8) as in Fig. 1b. This gives $\bar{a}$ good stability margin. The culture was grown overnight, and was diluted before each experiment so that the initial concentration was $\mathrm{OD}_{650}=0.12$ in all cases. The sensor was calibrated at each experiment using a spectrophotometer (Zuzi 4140) to compensate for absorbance of the medium.

To estimate the specific growth rate $\hat{\mu}$ from biomass measurements $x$ we used the super-twisting based observer (26) presented in [36]:

$$
\begin{aligned}
\sigma & =p^{-1} \log \left(\frac{x}{z_{1}}\right) \\
\dot{z}_{1} & =-D(t) z_{1}+p z_{1} z_{2}+2 p \beta|\sigma|^{\frac{1}{2}} \operatorname{sign}(\sigma) \\
\dot{z}_{2} & =\alpha \operatorname{sign}(\sigma) \\
\hat{\mu} & =p z_{2}
\end{aligned}
$$

The observer parameters were set to $\alpha=1.625, \beta=1.5$ and $p=0.2$ using the tuning methodology in [33], and its initial conditions were set to $z_{1}(0)=0.1, z_{2}(0)=1.25$. These result in $\hat{\mu}(0)=0.25$ for the estimated specific growth rate. We did not allow for an initial open-loop period for the observer to converge before closing the loop. This observer converges in finite time. In all cases it converged in less than one hour, as shown in Fig. 10 (middle).

Fig. 9 shows the experimental results fit well with the theoretical predictions considering the noisy, uncertain and timevarying context. We used the parameters $(a, b)=(4,7.11)$ corresponding, in the $\tau$ scale, to peak time $\tau_{p}=1.8$, settling time $\tau_{s}=2(98 \%$ criterium) and overshoot $\delta=0.03$, and $(a, b)=(2,4)$ for $\tau_{p} \approx 1.8, \tau_{s}=4$ and $\delta=0.16$. The proposed control law is compared with the baseline controller corresponding to (2) with $\gamma=1 / x^{\star}$. The comparison between the time responses in the $\tau-$ and $t-$ scales shows that order and magnitude are preserved. The experimental relationship between both time scales is shown in the bottom panel. As
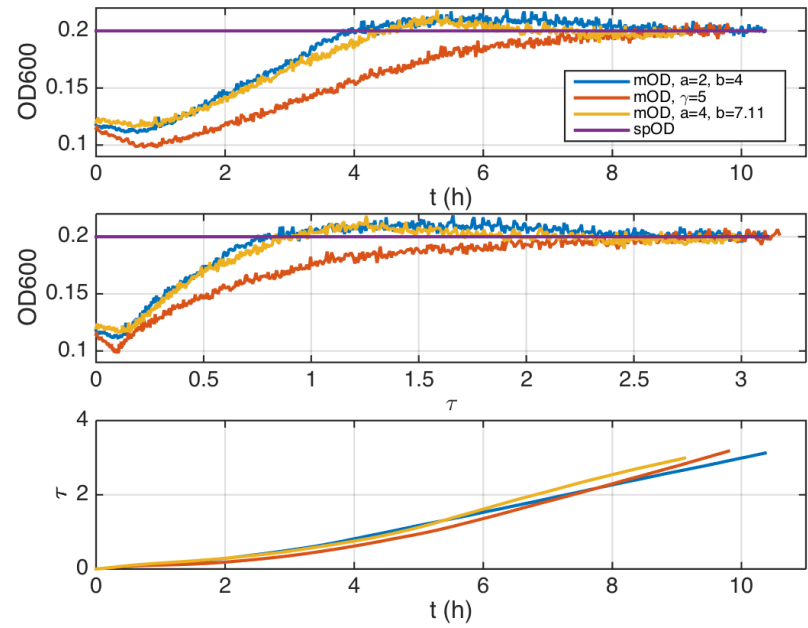

Figure 9. Biomass concentration $x$ in the $t$ time scale (top), in the transformed $\tau$-time (middle), and relationship between $t$ and $\tau$ (bottom). Initial conditions: $x_{0} \approx 0.12\left(O D_{600}\right)$. Set-point $x^{*}=0.2$. Proposed controller: $(a, b)=$ $(4,7.11)$ (yellow), $(a, b)=(2,4)$ (blue). Baseline controller (orange).

predicted by the simulations in section $\mathrm{V}$, although the $t(\tau)$ mappings are not the same for all cases they are very similar.

Fig. 10 shows the experimental dilution $D$, estimated specific growth rate $\hat{\mu}$ and controller gain $\gamma$. Notice the controller is robust with respect to uncertain factors that affect the specific growth rate and may differ from one experiment to another (e.g. substrate initial concentration, oxygen diffusion, cells metabolic state, etc.). This is reflected in the slightly different steady state value of the specific growth rate reached for each experiment. Notice there were performed independently in different days, so the environmental conditions (e.g.temperature) were most probably different. The controller designed in $\tau$ to achieve longer settling time $\left((a, b)=(2,4), \tau_{s}=4\right)$ indeed delivers lower values of dilution rate $D$ and has lower values of the adaptation gain $\gamma$ during the transient.

\section{CONCLUSION}

In this work we have proposed an adaptive control law that globally stabilizes the desired biomass set-point in continuous bioreactions. Using time scaling we render the system linear in the transformed time scale, where analysis and tuning of the controller becomes extremely simple. Stability is preserved in the original time domain. Important time-response characteristics such as order and magnitude relationships are also preserved in practice. Furthermore, our controller only assumes biomass concentration is measured, and does not require a detailed model of the growth kinetics or knowledge of the bioreaction yields. The intrinsic integral action of the gain adaptation rejects parameter uncertainties. In case the reaction rate is indirectly measured or calculated and some error appears, then the error is bounded, and the proposed controller can be tuned so as to significantly reduce it.

The simulation and experimental results validate the easiness to tune the controller to achieve desired time response 

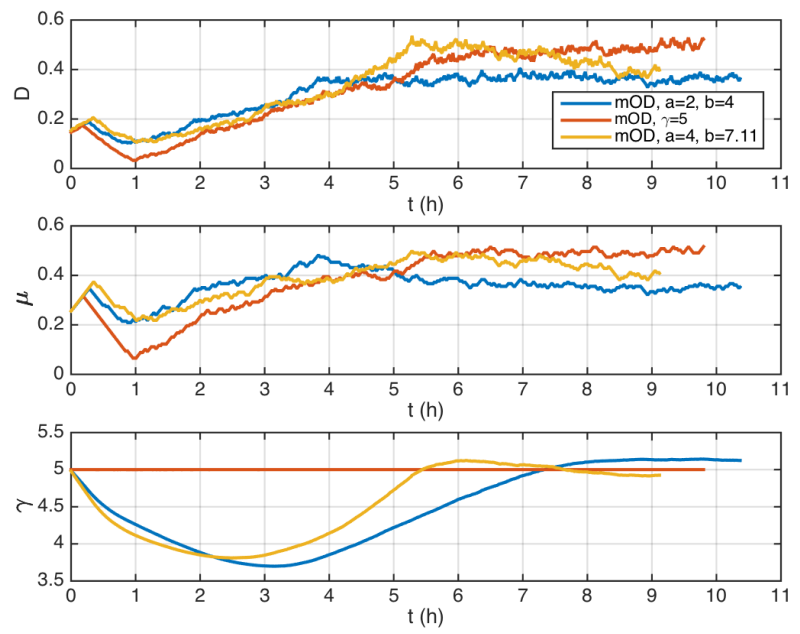

Figure 10. Dillution $D$ (top), estimated specific growth rate $\mu$ (middle) and controller gain $\gamma$ (bottom). Proposed controller: $(a, b)=(4,7.11)$ (yellow), $(a, b)=(2,4)$ (blue). Baseline controller (orange).

patterns, and its robustness in face of noisy uncertain bioreaction environments.

\section{REFERENCES}

[1] G. Bastin and D. Dochain, On-line Estimation and Adaptive Control of Bioreactors. Elsevier, 1990.

[2] H. De Battista, J. Picó, and E. Picó-Marco, "Nonlinear pi control of fedbatch processes for growth rate regulation," Journal of Process Control, vol. 22, no. 4, pp. 789-797, 42012.

[3] A. Schaum, J. Alvarez, and T. Lopez-Arenas, "Saturated pi control of continuous bioreactors with haldane kinetics," Chemical Engineering Science, vol. 68, pp. 520-529, 2012.

[4] F. Mazenc, J. Harmand, and M. Malisoff, "Stabilization in a chemostat with sampled and delayed measurements and uncertain growth functions," Automatica, vol. 78, pp. 241-249, 2017.

[5] J. Raftery, M. DeSessa, and M. Karim, "Economic improvement of continuous pharmaceutical production via the optimal control of a multifeed bioreactor," Biotechnol. Prog., vol. 33, no. 4, pp. 902-912, 2017.

[6] M. Scott, C. Gunderson, E. Mateescu, Z. Zhang, and T. Hwas, "Interdependence of cell growth and gene expression: Origins and consequences," Science, vol. 330, pp. 1099-1102, 2010.

[7] A. Saldanha, M. Brauer, and D. Botstein., "Nutritional homeostasis in batch and steady-state culture of yeast." Molecular Biology of the Cell, vol. 15, pp. 4089-4104, 2014.

[8] C. Gómez-Pérez and J. Espinosa, "The analysis of continuous bioreactors in series with recirculation using singular value decomposition," Chemical Engineering Research and Design, vol. 125, pp. 108-118, 2017.

[9] G. Kleman, J. Chalmers, G. Luli, and W. Strohl., "Glucose-stat, a glucose-controlled continuous culture," Applied and Environmental Microbiology, vol. 57, pp. 918-923, 1991.

[10] G. Szederkényi, N. Kristensen, K. hangos, and S. Jorgensen, "Nonlinear analysis and control of a continuous fermentation process," Computers and Chemical Engineering, vol. 26, pp. 659-670, 2002.

[11] M.-F. Jang, Y.-J. Chern, and Y.-S. Chou, "Robust adaptive controller for continuous bioreactors," Biochemical Engineering Journal, vol. 81, pp. $136-145,2013$

[12] G. Lara-Cisneros, J. Alvarez-Ramirez, and R. Femat, "Self-optimising control of a class of continuous bioreactor via variable-structure feedback," Int. J. of Systems Science, vol. 47, no. 6, pp. 1394-1406, 2016.

[13] N. Marcos, M. Guay, and D. Dochain, "Output feedback adaptive extremum seeking control of a continuous stirred tank bioreactor with monod's kinetics," Journal of Process Control, vol. 14, pp. 807-818, 2004.

[14] L. Mailleret, O. Bernard, and J.-P. . P. Steyer, "Nonlinear adaptive control for bioreactors with unknown kinetics," Automatica, vol. 40, no. 8, pp. 1379-1385, 2004.
[15] G. Savoglidis and C. Kravaris, "Constant-yield control of continuous bioreactors," Chemical Engineering Journal, vol. 228, pp. 1234-1247, 2013.

[16] K. S. Lee, P. Boccazzi, A. J. Sinskey, and R. J. Ram, "Microfluidic chemostat and turbidostat with flow rate, oxygen, and temperature control for dynamic continuous culture." Lab Chip, vol. 11, no. 10, pp. 1730-9, 52011.

[17] C. N. Takahashi, A. W. Miller, F. Ekness, M. J. Dunham, and E. Klavins, "A low cost, customizable turbidostat for use in synthetic circuit characterization." ACS Synth Biol, 72014.

[18] J. de Vree, R. Bosma, R. Wieggers, S. Gegic, M. Janssen, M. Barbosa, and R. Wijffels., "Turbidostat operation of outdoor pilot-scale photobioreactors," Algal Research, vol. 18, pp. 198-208, 2016.

[19] A. Bolic, H. Larsson, S. Hugelier, A. Lantz, U. Krahne, and K. Gernaey, "A flexible well-mixed milliliter-scale reactor with high oxygen transfer rate for microbial cultivations," Chemical Engineering J., vol. 303, pp. 655-666,, 2016.

[20] A. Milias-Argeitis, M. Rullan, S. Aoki, P. Buchmann, and M. Khammash, "Automated optogenetic feedback control for precise and robust regulation of gene expression and cell growth," Nature Communications, vol. 7:12546, pp. 1-11, 2016

[21] S. Hoffmann, C. Wohltat, K. Muüller, and K. M. Arndt, "A user-friendly, low-cost turbidostat with versatile growth rate estimation based on an extended kalman filter," PLoS ONE, vol. 12, no. 7:e0181923, pp. 1-15, 2017.

[22] J. Qiu, M. Arnold, and D. Murhammer, "On-line near infrared bioreactor monitoring of cell density and concentrations of glucose and lactate during insect cell cultivation," Journal of Biotechnology, vol. 173, pp. 106-111, 2014

[23] R. Fan, M. Ebrahimi, H. Quitmann, M. Aden, and P. Czermak, "An innovative optical sensor for the online monitoring and control of biomass concentration in a membrane bioreactor system for lactic acid production," Sensors, vol. 16, no. 3, pp. 411-423, 2016.

[24] B. Downey, L. Graham, J. Breit, and N. Glutting., "A novel approach for using dielectric spectroscopy to predict viable cell volume (vcv) in early process development," Biotechnology Progress, vol. 30, pp. 479487, 2014.

[25] V. Konakovsky, A. Yagtu, C. Clemens, M. Müller, M. Berger, S. Schlatter, and C. Herwig, "Universal capacitance model for real-time biomass in cell culture," Sensors, vol. 15, pp. 22 128-22 150, 2015.

[26] I. Dunn, E. Heinzle, J. Ingham, , and J. Přenosil, Biological Reaction Engineering: Dynamic Modelling Fundamentals with Simulation Examples, Second Edition. Wiley-VCH Verlag, 2003.

[27] M. Perrier and D. Dochain, "Evaluation of control strategies for anaerobic digestion processes," International Journal of Adaptive Control and Signal Processing, vol. 7, no. 4, pp. 309-321, 1993.

[28] T. Proll and N. Karim, "Nonlinear control of a bioreactor model using exact and I/O linearization," International Journal of Control, vol. 60 , no. 4, pp. 499-519, 1994

[29] G. Bastin and J. V. Impe, "Nonlinear and adaptive control in biotechnology: A tutorial." European J. of Control, vol. 1, no. 1, pp. 37-53, 1995.

[30] E. Picó-Marco, J. Picó, and H. De Battista, "Sliding mode scheme for adaptive specific growth rate control in biotechnological fed-batch processes," Int. J. of Control, vol. 78, no. 2, pp. 128-141, 2005.

[31] H. De Battista, J. Picó, and E. Picó-Marco, "Globally stabilizing control of fed-batch processes with haldane kinetics using growth rate estimation feedback," Journal of Process Control, vol. 16, no. 8, pp. 865-875, 9 2006.

[32] R. Antonelli and A. Astolfi, "Nonlinear controllers design for robust stabilization of continuous biological reactors," in Control Applications, 2000. Proceedings of the 2000 IEEE International Conference on. IEEE, 2000, pp. 760-765.

[33] J. Picó, E. Picó-Marco, A. Vignoni, and H. De Battista, "Stability preserving maps for finite-time convergence: Super-twisting slidingmode algorithm," Automatica, vol. 49, no. 2, pp. 534-539, 22013.

[34] A. Vargas, J. A. Moreno, and A. Vande Wouwer, "A weighted variable gain super-twisting observer for the estimation of kinetic rates in biological systems," Journal of Process Control, vol. 24, no. 6, pp. $957-$ 965, 62014.

[35] R. Milo, R. Phillips, and N. Orme, Cell Biology by the Numbers. Garland Science, 2016.

[36] H. De Battista, J. Picó, F. Garelli, and J. L. Navarro, "Reaction rate reconstruction from biomass concentration measurement in bioreactors using modified second-order sliding mode algorithms." Bioprocess Biosyst Eng, vol. 35, no. 9, pp. 1615-25, 2012. 